\title{
A rare presentation of isolated sixth nerve palsy as dolichoectasia of vertebral artery
}

\begin{abstract}
Vertebrobasilar dolichoectasia is a condition where the vertebral arteries are dilated, elongated and distorted, causing significant deterioration of tunica intima. The most common causes of sixth nerve palsy include infection, stroke, brain tumour and injury. We reported a case of a 56-year-old man with underlying of diabetes mellitus and hypertension who presented with diplopia over his left gaze. There are no other associated neurology signs. Computed tomography (CT) brain showed well-defined hypodensities at right lentiform nucleus and brain magnetic resonance angiography (MRA) showed impingement of V4 vertebral artery to anterior lower pons near midline which is the exit of the left abducens nerve from the pons. This case illustrated the importance to investigate thoroughly the causes of sixth cranial nerve palsy.
\end{abstract}

Keywords: dolichoectasia, vertebral artery, isolated sixth nerve palsy, vertebrobasilar dolichoectasia
Volume II Issue 4 - 202 I

\author{
Choo Xin Wei,' Lim Wei Juan² \\ 'Department of Medicine, Hospital Sultanah Aminah Johor \\ Bahru, Malaysia \\ ${ }^{2}$ Department of Cardiology, National Heart Institute, Malaysia
}

Correspondence: Lim Wei Juan, Department of Cardiology, National Heart Institute, Kuala Lumpur, Malaysia, Tel 6097738765,Email omegakimia@yahoo.com

Received: August 01, 202I | Published: August 12, 2021
Abbreviations: CISS, constructive interference in steady state; CPA, cerebellopontine angle; CT: computed tomography; FIESTA, fast imaging employing steady state acquisition; MLF, medial longitudinal fasciculus; MRA, magnetic resonance angiography; WI, weighted imaging; VBD, vertebrobasilar dolichoectasia

\section{Introduction}

Vertebrobasilar dolichoectasia is a condition where the vertebral arteries are dilated, elongated and distorted, causing significant deterioration of tunica intima. Common causes of sixth nerve palsy include infections, stroke, brain tumour and traumatic brain injury. It is mostly associated with multiple vascular risk factors such as hypertension, dyslipidaemia and smoking and usually affects middleaged men. Dolichoectasia usually affects basilar and carotid arteries and it rarely involved vertebral artery which is usually asymptomatic. The most common outcome of vertebral artery dolichoectasia is mainly transient ischemic attack, ischemic stroke and intracerebral haemorrhage and is always associated with facial weakness and pyramidal signs due to sixth nerve palsy. Brain magnetic resonance imaging (MRI) is essential to illustrate the vertebral artery dolichoectasia. We reported a 56-year old male who presented to us with horizontal diplopia and left abducens nerve palsy which were relieved by antihypertensive therapy.

\section{Case report}

A 56-year-old man who is an active smoker, with underlying of hypertension and diabetes, presented with 2 days history of painless binocular horizontal double vision and giddiness. Diplopia was more prominent over left gaze. Otherwise, there was no blurring of vision, eye pain, fever, headache, seizures and previous trauma over his head. He also denied of having constitutional symptoms to suggest malignancy such as loss of appetite and loss of weight. He was discharged 3 weeks ago from Hospital Sultanah Aminah for hypertensive urgency. During that admission, his blood pressure was $219 / 113 \mathrm{mmHg}$, he was started with tablet amlodipine 10mg daily. He has been compliance to medications.

Upon arrival to Emergency Department, his measured blood pressure was 202/106mmHg. His ophthalmologic examination revealed horizontal nystagmus over right eye and incomplete left eye abduction which is not corrected by Doll's eye manoeuvre. There was no involvement of other cranial nerves which suggestive of cavernous sinus syndrome or cerebellopontine (CPA) angle lesion. Physical examination also did not reveal signs of upper motor neuron lesion or cerebellar signs. There were no lymph nodes palpable and there was no mastoid tenderness to suggest Gradenigo's syndrome. There was no evidence of papilledema with fundoscopy examination. There were no thyroid eye signs or fatiguability to suggest myasthenia gravis.

All other blood investigations including thyroid function tests and autoimmune screening were within normal limits. Plain computed tomography (CT) brain was done and revealed welldefined hypodensities seen in right lentiform nucleus chronic infarct. ${ }^{1}$ Otherwise, no intracranial haemorrhage and no acute ischemic infarcts seen at other areas. Brain magnetic resonance angiography (MRA) was ordered and it showed impingement of V4 vertebral artery to anterior lower pons near midline which is the exit of the left abducens nerve from the pons and also trigeminal nerve (blue arrow) and right abducens nerve (Figure 1-3) No abnormal signals were seen in brainstem. After ruling out other aetiologies of sixth cranial nerve, diagnosis of isolated sixth cranial nerve palsy was made.

\section{Treatment and outcome}

Patient was managed conservatively by controlling his blood pressure and diabetes mellitus with tablet amlodipine 10mg daily, tablet perindopril $4 \mathrm{mg}$ daily and metformin. His blood pressure has been able to control within $110-130 / 60-80$ throughout his stay. His diplopia was also resolved after two days. 


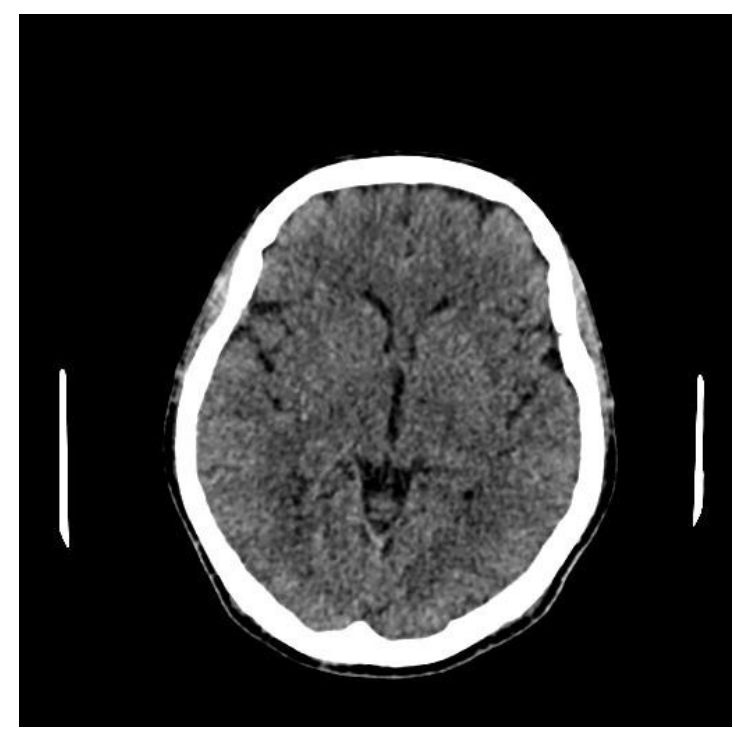

Figure I CT brain showing right lentiform nucleus chronic infarct.

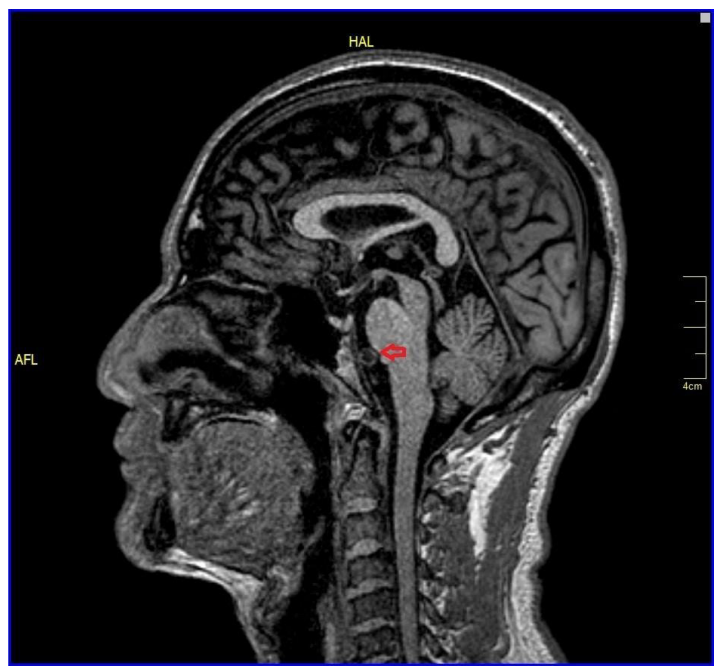

Figure 2 Brain magnetic resonance angiography (MRA) showing impingement of $\mathrm{V} 4$ vertebral artery (red arrow) to anterior lower pons near midline which is the exit of the left abducens nerve from the pons.

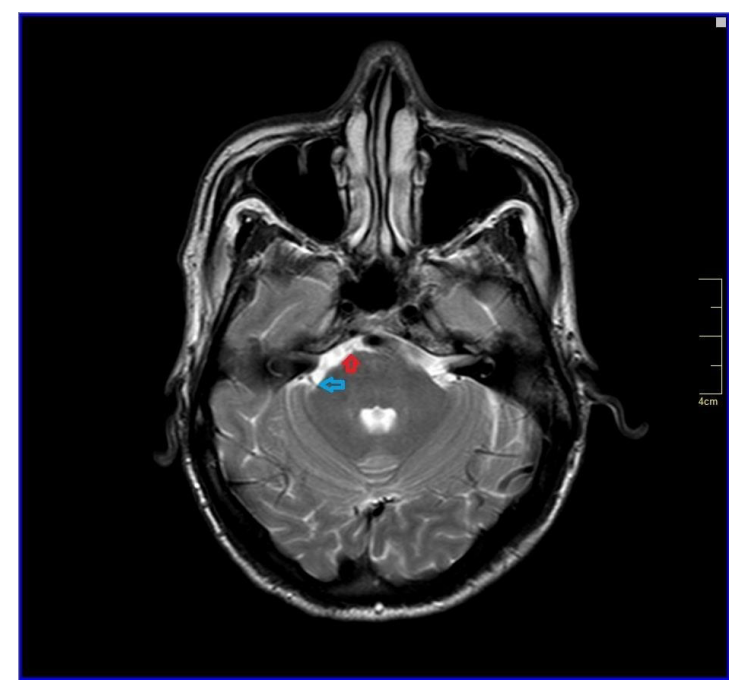

Figure 3 Brain magnetic resonance angiography (MRA) showing trigeminal nerve (blue arrow) and right abducens nerve (red arrow).
Patient was followed up after 6 weeks with unchanged left abduction defect, his blood pressure and diabetes were well controlled with oral medications.

\section{Discussion}

Sixth cranial nerve also known as the Abducens nerve, innervates the ipsilateral lateral rectus muscle of the eye to abduct the ipsilateral eye. The sixth cranial nerve has a long subarachnoid course and it is located in the pons, just ventral to the floor of the fourth ventricle and just lateral to the medial longitudinal fasciculus (MLF). ${ }^{1}$

Sixth cranial nerve palsy secondary to dolichoectasia of vertebral artery is a rare but important diagnosis to consider after rule out other differential diagnosis. It requires MRI study preferably using constructive interference in steady state (CISS) imaging technique to visualize the entire course of sixth cranial nerve. ${ }^{2}$ The pathogenesis of intracranial artery dolichoectasia is remain unclear. However, studies reported multiple pathophysiological processes that might contribute to 6th cranial nerve palsy, including systemic hypertension, diabetes mellitus and atherosclerosis. ${ }^{3}$

In our case, patient presented with solely left diplopia due to isolated sixth cranial nerve palsy due to vertebrobasilar dolichoectasia (VBD) with no other associated clinical symptoms. Asymptomatic VBD occurred in $90 \%$ of the patients. ${ }^{4}$ Other symptoms that were reported included cerebellar dysfunction, brainstem syndrome, ischemic stroke and trigeminal neuralgia. ${ }^{5-7}$ For example, Madhugiri et al described a patient who presented with diplopia, ipsilateral facial pain and facial muscle twitches, attributed to compression of the abducens, trigeminal and facial nerves caused by VBD, respectively. ${ }^{8}$ Pham et al reported a case of VBD resulting in both abducens and ipsilateral trigeminal nerve palsies. ${ }^{9}$

Radiological examination with $\mathrm{CT}$ and MRI/MRA brain is important to look for the causes of sixth cranial nerve palsy and to understand the underlying pathology. King et al reported spontaneous recovery rate of the abducent nerve seemed to be lower if the cause is due to vascular compression than that with an undetermined etiology. ${ }^{10,11}$ Hence, a plain MRI/ MRA brain might not be able to clearly detect the compressed abducens nerve. MRI/MRA with heavy T2 weighted imaging (WI), constructive interference in steady state (CISS), and fast imaging employing steady state acquisition (FIESTA) are essential to demonstrate vascular compression of the abducent nerve. ${ }^{12}$ In our case, MRI (CISS) was done and there was no neurovascular compression of the Abducens nerve. This could potentially explained the fast recovery from the sixth cranial nerve palsy.

Prognosis for most of the cases of VBD causing sixth cranial nerve palsies are usually favourable with most of them remaining stable. ${ }^{13}$ In our case, patient did not have any complications or deterioration of symptoms. He continued his follow up for hypertension and diabetes to control the risk factor.

\section{Conclusion}

Though isolated 6th nerve palsy is the most common oculomotor nerve palsy, the natural history of abducent nerve palsy may be detected using MRI with CISS or FIESTA. Conservative management such as strict blood pressure control is important to control the symptoms.

\section{Acknowledgments}

We would like to acknowledge Dr Hamdi, our consultant neurologist for helping us with the nerve conduction study. 


\section{Funding}

None.

\section{Conflicts of interest}

There is no conflict of interest in this case report.

\section{References}

1. Kline LB. Neuro-ophthalmology Review Manual. 6th ed. SLACK Inc; 2008.

2. Jeeva-Patel T, Margolin EA, Mandell D. Sixth cranial nerve palsy secondary to compression by dolichoectatic vertebrobasilar artery. $B M J$ Case Rep. 2020;6:13(7):e234949.

3. Svien HJ, Peserico L. Occlusion of the third ventricle by tortuous, bulbous, calcified basilar artery. Neurology. 1959;9:836-838.

4. Resta M, Gentile MA, Di Cuonzo F et al. Clinical-angiographic correlations in 132 patients with megadolichovertebrobasilar anomaly. Neuroradiology. 1984;26(3):213-216.

5. Ubogu EE, Zaidat OO. Vertebrobasilar dolichoectasia diagnosed by magnetic resonance angiography and risk of stroke and death: A cohort study. J Neurol Neurosurg Psychiatry. 2004;75(1):22-26.
6. Milandre L, Bonnefoi B, Pestre $\mathrm{P}$ et al. Vertebrobasilar arterial dolichoectasia. Complications and prognosis. Rev Neurol. 1991;147(11):714-722.

7. Tomasello F, Alafaci C, Salpietro FM et al. Bulbar compression by an ectatic vertebral artery: A novel neurovascular construct relieved by microsurgical decompression. Neurosurgery. 2005;56(1 Suppl):117-124.

8. Madhugiri VS, Roopesh Kumar VR, Gopalakrishnan MS, et al. Cranial polyneuropathy associated with vertebrobasilar dolichoectasia. Clin Neurol Neurosurg. 2012;114(7):1059-1061.

9. Pham T, Wesolowski J, Trobe JD. Sixth cranial nerve palsy and ipsilateral trigeminal neuralgia caused by vertebrobasilar dolichoectasia. Am J Ophthalmol Case Rep. 2018;2:10:229-232.

10. King AJ, Stacey E, Stephenson G, et al. Spontaneous recovery rates for unilateral sixth nerve palsies. Eye (Lond). 1995;9(Pt 4):476-478.

11. Ohhashi $\mathrm{G}$, Irie $\mathrm{K}$, Tani $\mathrm{S}$ et al. Isolated abducens nerve palsy caused by the compression of the basilar artery: A case report. No To Shinkei. 2001;53(1):69-72.

12. Narai H, Manabe Y, Deguchi K, et al. Isolated abducens nerve palsy caused by vascular compression. Neurology. 2000;8:55(3):453-454.

13. Kato H, Nakajima M, Ohnaka Y, et al. Recurrent abducens nerve palsy associated with neurovascular compression. J Neurol Sci. 2010;15:295(12):135-136. 